\title{
The Local Group Census: Planetary nebulae in Sextans B ${ }^{\star}$
}

\author{
L. Magrini ${ }^{1}$, R. L. M. Corradi ${ }^{2}$, N. A. Walton ${ }^{2}$, A. A. Zijlstra ${ }^{3}$, D. L. Pollacco ${ }^{4}$, \\ J. R. Walsh ${ }^{5}$, M. Perinotto ${ }^{1}$, D. J. Lennon ${ }^{2}$, and R. Greimel ${ }^{2}$
}

1 Dipartimento di Astronomia e Scienza dello Spazio, Universitá di Firenze, L.go E. Fermi 2, 50125 Firenze, Italy

2 Isaac Newton Group of Telescopes, Apartado de Correos 321, 38700 Santa Cruz de La Palma, Canarias, Spain

3 Physics Department, UMIST, PO Box 88, Manchester M60 1QD, UK

4 School of Pure and Applied Physics, Queen's University Belfast, Belfast BT7 9NN, Northern Ireland, UK

5 ST-ECF, E.S.O., Karl-Schwarzschild-Strasse 2, 85748 Garching bei München, Germany

Received 21 December 2001 / Accepted 26 February 2002

\begin{abstract}
Five planetary nebulae (PNe) have been discovered in the nearby dwarf irregular galaxy. Emission line images were obtained using the Wide Field Camera of the $2.5 \mathrm{~m}$ Isaac Newton Telescope (INT) at La Palma (Spain). The candidate PNe were identified by their point-like appearance and relatively strong [O III] emission-line fluxes. They are located within a galactocentric distance of 2.8 arcmin, corresponding to $1.1 \mathrm{kpc}$ at the distance of Sextans B. Luminosities are in the range 1800-5600 $L_{\odot}$. Sextans B is one of the smallest dwarf irregular galaxies with a PN population. The number of PNe detected suggest an enhanced star formation rate between 1 and 5 Gyr ago.
\end{abstract}

Key words. planetary nebulae: individual - galaxies: individual: Sextans B

\section{Introduction}

The galaxies of the Local Group (LG) are our closest neighbors in the Universe. All the galaxies within $1.25 \mathrm{Mpc}$ from its barycentre are considered members of the LG (Courteau \& van den Bergh 1999). The LG contains galaxies covering a large range of luminosities, metallicities, and morphological types, but the large majority are dwarf irregular and spheroidal galaxies. Their close distance enables the study of their content in much more detail than in more distant galaxies. For this reason, a new deep survey, the Local Group Census ( $\mathrm{LGC}^{1}$ ) has been undertaken in all the LG galaxies above $\mathrm{Dec}=-30^{\circ}$. The survey aims at highlighting all populations with strong emission lines, such as, H II regions, planetary nebulae $(\mathrm{PNe})$, supernova remnants, luminous blue variables, and Wolf-Rayet stars.

One of the first targets of the LGC was the dwarf irregular galaxy Sextans B, which at a distance of $1.32 \pm 0.12 \mathrm{Mpc}$ (Sakai et al. 1997) is located at the outer fringes of the Local Group. Although its radial velocity

Send offprint requests to: R. Corradi,

e-mail: rcorradi@ing.iac.es

* Based on observations obtained at the $2.5 \mathrm{~m}$ INT telescope operated on the island of La Palma by the Isaac Newton Group in the Spanish Observatorio del Roque de Los Muchachos of the Instituto de Astrofisica de Canarias.

1 See http://www.ing.iac.es/rcorradi/LGC is consistent with membership of the LG, it might form, together with NGC 3109, Antlia, Sextans A and perhaps AM1013-394A, a physical association of galaxies lying just beyond the boundaries of the LG, which would be the nearest association outside the LG (van den Bergh 2000). The resolved stellar population of Sextans B is dominated by red giants (Sakai et al. 1997), with a prominent contribution of intermediate-age asymptotic branch stars. A young population is also present, indicating a complex, low-rate and likely discontinuous star formation throughout the galaxy lifetime (Tosi et al. 1991; van den Bergh 2000). A dozen H II regions are known (Strobel et al. 1991), but no PNe have been identified prior to this study (Jacoby \& Lesser 1981).

The narrow-band images ([O III], $\mathrm{H} \alpha+[\mathrm{N}$ II $]$, [S II], $\mathrm{He}$ II) presented in this paper show five new candidate $\mathrm{PNe}$ in Sextans B. The observations are described in Sect. 2, and data reduction and analysis in Sect. 3. In Sect. 4, we present the candidate $\mathrm{PNe}$, and discuss the results in Sect. 5.

\section{Observations}

We observed Sextans B (DDO 70, 10h00m 00s $+05 \mathrm{~d} 19 \mathrm{~m} 56 \mathrm{~s}$, J2000.0) using the prime focus wide field camera (WFC) of the $2.54 \mathrm{~m}$ Isaac Newton Telescope (La Palma, Spain), on February and April 2001. The panoramic detector of the WFC consists of four thinned 
EEV CCDs of $2048 \times 4096$ pixels each, and with a pixel scale of $0^{\prime \prime} 33$. The $34^{\prime} \times 34^{\prime}$ field of view of the camera covered the entire galaxy. Four narrow-band filters were used, with the following central wavelengths and full widths at half maximum $(F W H M \mathrm{~s})$ : [O $\mathrm{III}](500.8 / 10.0 \mathrm{~nm})$, $\mathrm{H} \alpha+[\mathrm{N}$ II] $(656.8 / 9.5)$, He II $(468.6 / 10.0)$ and [S II] (672.5/8.0). A Strömgren $y$ filter, centred at $555.0 \mathrm{~nm}$ and with a $F W H M$ of $30.0 \mathrm{~nm}$, was also used to obtain off-band images for continuum subtraction.

Exposure times were $600 \mathrm{~s}$ for $y, 1200 \mathrm{~s}$ for [O III] and $\mathrm{H} \alpha+[\mathrm{N} \mathrm{II}]$, and $3600 \mathrm{~s}$ for He II, and [S II], (split into three sub-exposures). The seeing was $1^{\prime \prime}$ through the [O III], $\mathrm{H} \alpha+[\mathrm{N} \mathrm{II}],[\mathrm{S} \mathrm{II}]$ and $y$ filters, and 1".5 through the He II filter. Several observations were made each night of the spectrophotometric standard stars: BD +33 2642, G191-B2B and Feige 34 (Oke 1990).

\section{Data reduction and analysis}

The frames were processed using IRAF. They were debiased and flat-fielded using the ING WFC data-reduction pipeline (Irwin \& Lewis 2001). Subsequently, they were corrected for geometrical distortions and aligned to the [O III] frames. The sky background was determined in areas far from the galaxy and then subtracted from all images.

Emission-line objects were identified using a standard on-band/off-band technique (Magrini et al. 2000). Briefly, the stellar continuum of Sextans B (Strömgren $y$ images, properly scaled) was subtracted from the narrow-band images in order to highlight emission-line objects. Aperture photometry was applied using the IRAF task APPHOT in the continuum-subtracted frames. Photometric errors were derived from photon statistics for both sources and background with APPHOT. They are of few per cents both for $[\mathrm{O} \mathrm{III}]$ and for $\mathrm{H} \alpha+[\mathrm{N}$ II] fluxes.

The instrumental magnitudes were calibrated by convolving the spectrum of the standards used (Oke 1990) with the response curve of each filters. An interstellar extinction of $E(B-V)=0.02$ was used (de Vaucouleurs et al. 1991), following the Seaton (1979) prescription. The fluxes in the [O $\mathrm{III}]$ line at $\lambda=500.7 \mathrm{~nm}$ were corrected for the contribution of the companion oxygen line at $\lambda 495.9 \mathrm{~nm}$, which amounts to $20 \%$ of the total flux in the relatively broad filter used. Including the uncertainties in all steps, we estimate that the total absolute error on the absolute fluxes quoted in this paper is $\leq 20 \%$.

The astrometric solution for the [O III] frames was also obtained through the WFC pipeline using the USNO A2.0 catalogue (Monet et al. 1998). The solution has an rms accuracy of $0^{\prime \prime} 3$.

\section{Candidate planetary nebulae}

The $\mathrm{H} \alpha+[\mathrm{N} \mathrm{II}]$ and [O III] continuum-subtracted images (Fig. 1, left panels) show a number of emission-line objects. Some of them are clearly extended H II regions, which were previously studied by Moles et al. (1990), Strobel et al. (1991), Hunter et al. (1993), Hunter \& Hoffman (1999), and Roye \& Hunter (2000). We note that these regions form an approximate ring, with another $\mathrm{H} \alpha$ nebulosity in the centre of the ring, perhaps close to the nucleus.

Candidate PNe in Sextans B were selected using the following criteria (Magrini et al. 2000, 2001):

i) they should appear both in the [O III] and $\mathrm{H} \alpha+[\mathrm{N} \mathrm{II}]$ images but not in the continuum frame;

ii) they should have a stellar point spread function: at the distance of Sextans B. PNe are normally $0.1-1$ pc in diameter, corresponding to 15-150 mas at the distance of Sextans B.

Five objects in Sextans B fulfill the criteria above; these new candidate $\mathrm{PNe}$ are listed in Table 1 and marked in Fig. 1. Only [O III] and $\mathrm{H} \alpha+[\mathrm{N} \mathrm{II}]$ fluxes are quoted in Table 1: they were not detected in He II and [S II]. The upper limit to these latter fluxes is estimated to be $10^{-16} \mathrm{erg} \mathrm{cm}^{-2} \mathrm{~s}^{-1}$. The [O III] fluxes were converted into equivalent $V$-band magnitudes following Jacoby (1989):

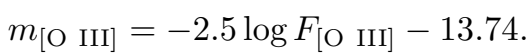

The luminosity was obtained from the relation $L \approx 150 \times$ $L(\mathrm{H} \beta)$ (Zijlstra \& Pottasch 1989), which is correct when the nebula is optically thick. This assumes that the [N II] lines make a negligible contribution to the $\mathrm{H} \alpha$ line, which is likely correct at this low metallicity. The luminosities are close to but below the value of $7000 L_{\odot}$, as expected for progenitor masses $<2 M_{\odot}$.

This is the first identification of PNe in Sextans B: previous surveys did not find any suitable candidate (Jacoby \& Lesser 1981). The five candidate $\mathrm{PNe}$ are distributed over an area of $1.5 \times 4.5$, corresponding to a linear, projected size of $0.6 \mathrm{kpc} \times 1.7 \mathrm{kpc}$. Three of them are found in regions densely populated by stars, whilst the other two (SexB PN1 and SexB PN5) are located in the outskirts of Sextans B, at projected distances from the galaxy centre of 0.8 and $1.1 \mathrm{kpc}$. All the candidate PNe have $[\mathrm{O}$ III $] /(\mathrm{H} \alpha+[\mathrm{N}$ II $])$ flux ratios between 1.4 and 2.3. These are typical values for Galactic PNe (cf. Magrini et al. 2000), although the oxygen abundance of Sextans B is only 0.16 times solar (Moles et al. 1990).

\subsection{Completeness limits}

The detection of the five PNe raises the question: how many more remain to be discovered? Some PNe could have been missed in the most crowded region of Sextans B. We have estimated this number by adding "artificial stars" within the range of luminosities expected for PNe (Jacoby 1989) in both the [O III] and the off-band, $y$, frames using the IRAF task ADDSTAR. These artificial stars were recovered following the same procedures used to detect PNe. A $3 \sigma$ detection limit was adopted in the off-band frame. The recovery rate is significantly lower in the continuumsubtracted frames because of the larger noise produced 

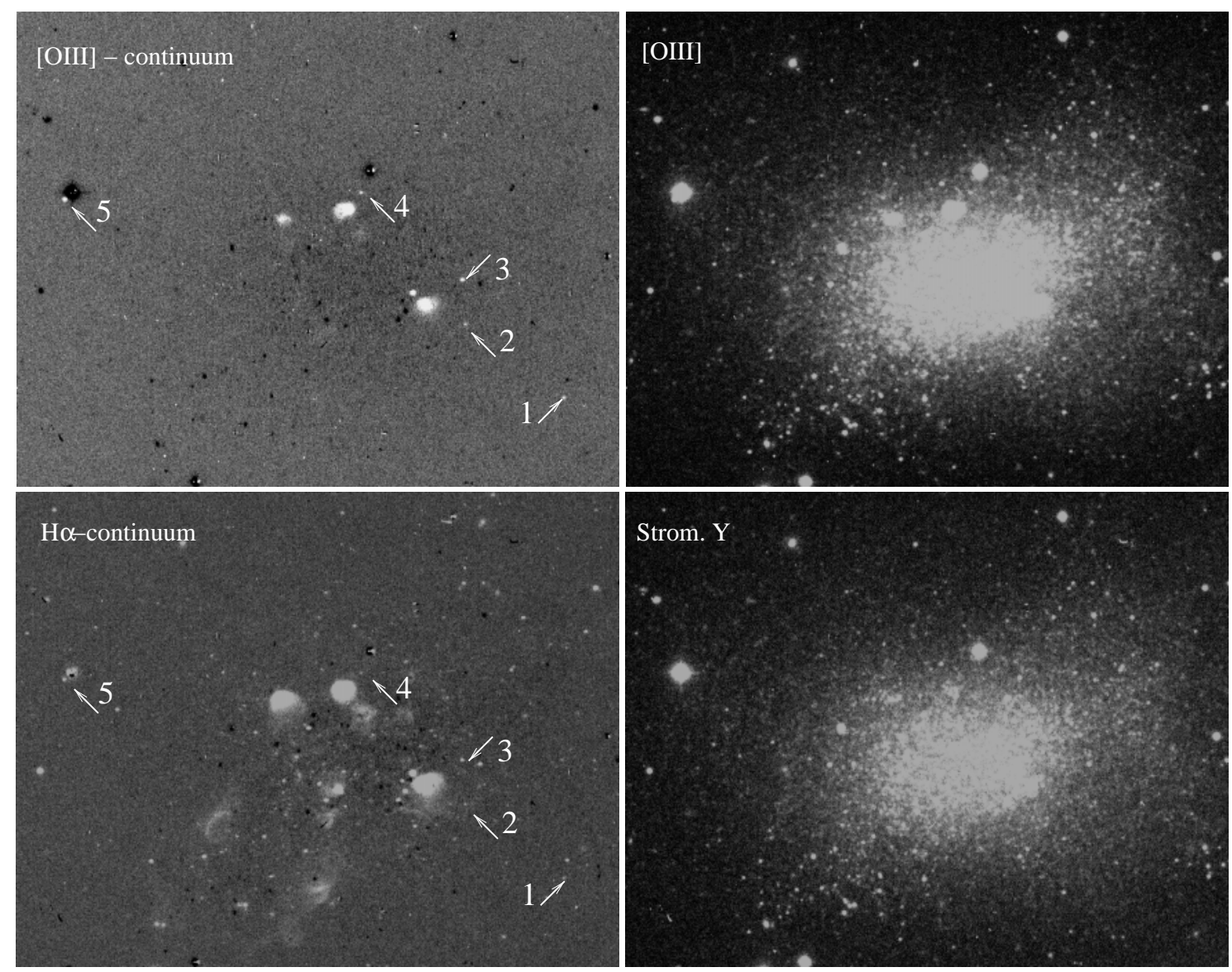

Fig. 1. INT + WFC images of Sextans B. The size of each frame is $55^{\prime} 1 \times 4.0$, i.e. a small fraction of the whole field of view of the WFC. North is at the top, East to the left. In the continuum subtracted images, candidate PNe are indicated by the arrows and marked with the identification number as given in Table 1.

Table 1. PN candidates in Sextans B. Positions and reddening corrected $\mathrm{H} \alpha+[\mathrm{N} \mathrm{II}]$ and $[\mathrm{O}$ III] emission-line fluxes (in units of $10^{-16} \mathrm{erg}^{-2} \mathrm{sm}^{-1}$ ) are given, and luminosity in solar units.

\begin{tabular}{lrrrrrl}
\hline \hline Identification & \multicolumn{2}{c}{ RA $(2000.0)$ Dec } & $F_{[\mathrm{OIII}]}$ & $F_{\mathrm{H} \alpha}$ & $m_{[\mathrm{O} \text { III }]}$ & $L$ \\
\hline SexB PN1 & 95953.06 & 51852.1 & 16.0 & 7.0 & 23.24 & 2000 \\
SexB PN2 & 95956.50 & 51929.5 & 11.2 & 8.1 & 23.64 & 2300 \\
SexB PN3 & 95956.64 & 51952.6 & 19.2 & 13.0 & 23.05 & 3600 \\
SexB PN4 & 100000.19 & 52037.2 & 12.8 & 6.6 & 23.49 & 1800 \\
SexB PN5 & 100010.48 & 52031.9 & 38.4 & 20.0 & 22.30 & 5600 \\
\hline
\end{tabular}

in the scaling and subtraction of two images. This effect is dominant in recovering emission-line objects at faint magnitudes.

The incompleteness is a combination of the probability of missing an object in the emission-line image and the probability of wrongly identifying a star in the continuum frame. Incompleteness is defined as a recovery rate of artificial stars less then 50\% (e.g. Minniti \& Zijlstra 1997). This was computed in different regions of Sextans B, and for a range of assumed magnitudes. We find that incompleteness occurs for emission-line objects fainter than $m_{\text {[O III }]}=24.5$, located within 1.5 from the centre of the galaxy.
Counting all stars brighter than this completeness limit, we find that the total luminosity of these stars in the inner regions is about $40 \%$ of the total. This agrees (roughly) with 3 of the $5 \mathrm{PNe}$ found in this region. As completeness is better than $50 \%$ in this area, we conclude that at most five $\mathrm{PNe}$ brighter than $m_{[\mathrm{OIII}]}=24.5$ may have been missed there.

\subsection{Total PNe population size}

The number of candidate PNe that we found in Sextans B is consistent with the expected population size for this galaxy, which can be estimated from stellar population 


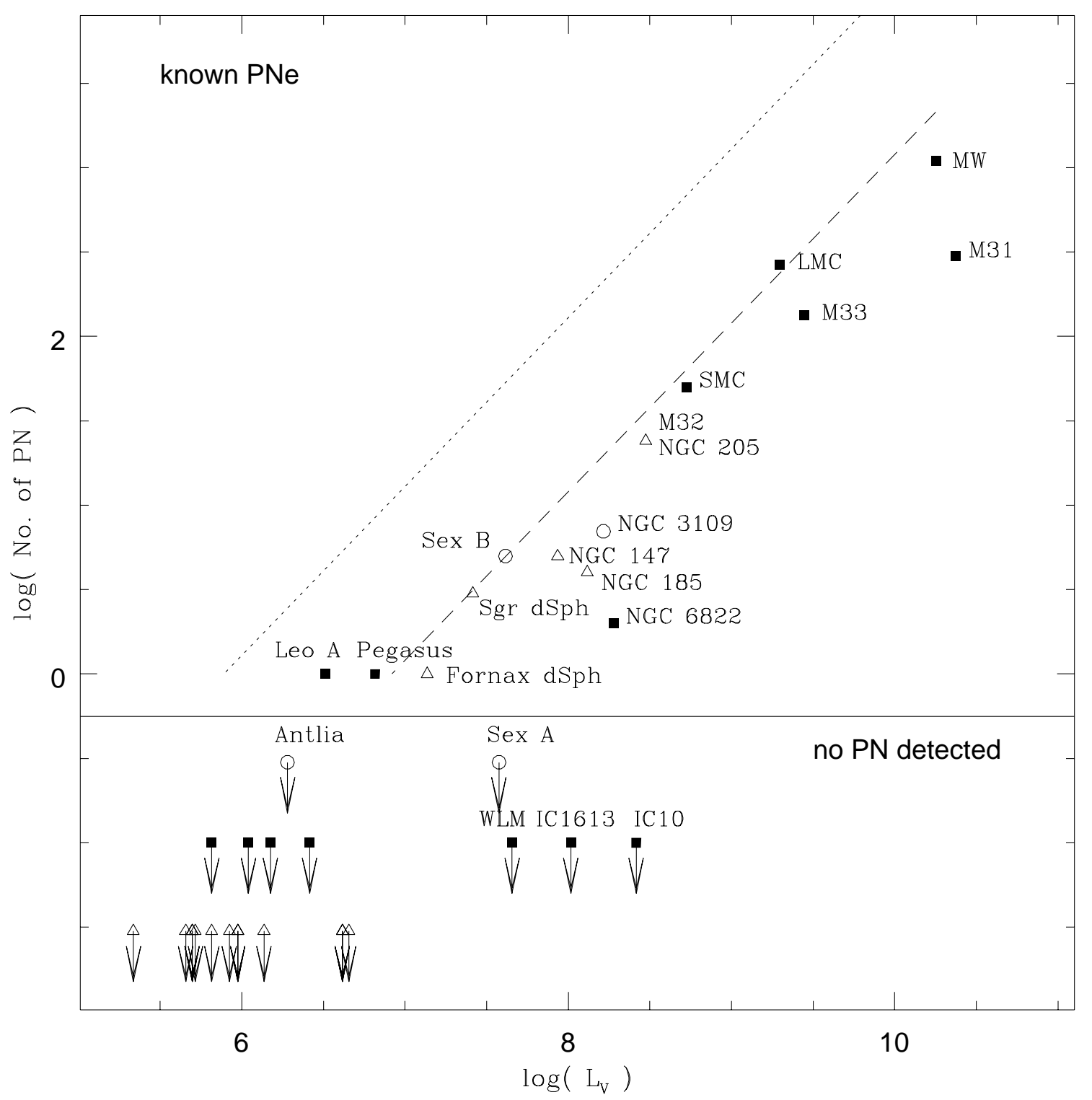

Fig. 2. The number of PNe of galaxies in or near the Local Group, versus the $V$-band luminosity in solar units. The dotted line shows the expected numbers based on a total number of PNe in the MW of 23000 . The dashed line is fitted to the known population in the LMC. Filled squares indicate LG gaseous galaxies, triangles indicate spheroidal galaxies and open circles show the NGC 3109 group.

models. For a simple (i.e. coeval and chemically homogeneous) stellar population, the number of stars $n_{j}$ in any post-main-sequence phase $j$ (Renzini \& Buzzoni 1986) is given by

$n_{j}=\dot{\xi} L_{\mathrm{T}} t_{j}$,

where $\dot{\xi}$ is the specific evolutionary flux (number of stars per unit luminosity leaving the main sequence each year), $L_{\mathrm{T}}$ the total luminosity of the galaxy, and $t_{j}$ the duration of the evolutionary phase $j$ ( $\leq 20000$ yrs for the PN phase). Adopting a bolometric luminosity of Sextans B of $\sim 10^{8} L_{\odot}$ (van den Bergh 2000), and a specific evolutionary flux between $5 \times 10^{-12}$ and $2 \times 10^{-11} \mathrm{yr}^{-1} L_{\odot}^{-1}$ (cf. Renzini \& Buzzoni 1986; Mendez et al. 1993), the corresponding population size of $\mathrm{PNe}$ in Sextans B is between 5 and 20 objects. This appears to be in good agreement with the number of $\mathrm{PNe}$ discovered. However, the [O III] luminosity of a $\mathrm{PN}$ rapidly declines once the nebulae becomes optically thin, and the phase during which a PN is bright enough to be discovered here is much shorter, typically 5000 yr (assuming an expansion velocity of $20 \mathrm{~km} \mathrm{~s}^{-1}$ and a radius of $0.1 \mathrm{pc}$ ). Thus, the number of PNe may be a little higher than expected.

Figure 2 shows the number of known PNe for all nearby galaxies, using data in van den Bergh (2000), with three exception: M 33 now has 131 known PNe (Magrini et al. 2001), whilst for the Sagittarius dwarf spheroidal $3 \mathrm{PNe}$ are now known (Walsh, priv. comm.), and for 
WLM both published PNe have been shown to be normal stars (Minniti \& Zijlstra 1997). For the Milky Way (MW), about $1100 \mathrm{PNe}$ are now known, but the total number has been estimated to be as high as 23000 (Zijlstra $\&$ Pottasch 1991). Figure 2 plots the number of PNe versus the $V$-band luminosity of the galaxy, in solar units. Equation (1) suggests that this relation should be strictly linear, for a uniform star formation history. The dashed line gives the expected total number of PNe scaling from 23000 in the MW. The dotted line is fitted to the known number in the LMC, where surveys have been most complete. It is clear that only the bright tip of the PN luminosity function can be detected. Figure 2 also shows that for galaxies with $M_{V}>-12.5$, one would not expect to detect the $\mathrm{PN}$ population.

Given its distance, the PN census in Sextans B may be expected to be less deep than that in the LMC. However, the two fall on the same line. This could be explained if Sextans B has a large proportion of intermediate-age stars, with a significant fraction of this star formation over the past 5 Gyr or so, compared to the LMC: a younger population has a higher death rate and will produce more $\mathrm{PNe}$. The star formation in the Universe has declined rapidly from its peak around $z \approx 1.5$ (Lilly et al. 1995; Madau et al. 1998); however, Sextans B may be an example of a galaxy with a more delayed star formation history. The distribution in Fig. 2, once completeness has been reached, can be used to measure the star formation history for intermediate-age stars.

The small population size prevents us from building a meaningful Planetary Nebulae Luminosity Function (PNLF) for Sextans B, a property that is generally used as an extragalactic distance indicator (Jacoby 1989). In fact, the absolute magnitude of the bright cutoff of the PNLF depends on the size of the PN population. For a small population as in Sextans B, the bright PNe defining the "universal" cutoff of the PNLF $\left(m_{[\mathrm{O} \text { III }]}^{\star}=-4.48\right.$, Jacoby 1989) are not observed owing to the poor sampling. The simulations of Mendez et al. (1993) show, that even for a population size of 500 PNe, typical of the LMC and much larger than in Sextans B, no PNe with absolute magnitude lower than -4.0 are expected. Therefore, the absolute magnitude of the brightest PN in Sextans B, equal to $-3.59 \mathrm{mag}$, is qualitatively consistent with its small population size, and can only provide an upper limit of $\sim 2 \mathrm{Mpc}$ for the distance of the galaxy.

Figure 2 and the completeness analysis above suggest that the PN census in Sextans B is now fairly complete. In many other galaxies (especially IC 10), significant PN populations remain to be found.

\section{Summary and conclusions}

Five new candidate $\mathrm{PNe}$ have been discovered in the dwarf irregular galaxy Sextans B. This is a notable result considering the limited number of $\mathrm{PNe}$ known in the other dwarf galaxies of the LG. In fact, a total of four PNe are known in the dwarf spheroidal companions of the Milky Way: three PNe in Sagittarius (Walsh et al. 1997; Dudziak et al. 2000), and one in Fornax (Danziger et al. 1978). Another few PNe have been discovered in the dwarf companions of M 31, and one in NGC 6822 (Ciardullo et al. 1989; Jacoby \& Ford 1986).

The number of candidate PNe detected in Sextans B is in good agreement with the PNe population size expected from stellar evolution models.

\section{References}

Ciardullo, R., Jacoby, G. H., Ford, H. C., \& Neil, J. D. 1989, ApJ, 339, 53

Courteau, S., \& van den Bergh, S. 1999, AJ, 118, 337

Danziger, I. J., Webster, B. L., Dopita, M. A., \& Hawarden, T. G. 1978, ApJ, 220, 458

de Vaucouleurs, G., de Vaucouleurs, A., Corwin, H. G., et al. 1991, Third Reference Catalog of Bright Galaxies (Berlin, Springer-Verlag)

Dudziak, G., Péquignot, D., Zijlstra, A. A., \& Walsh, J. R. 2000, A\&A, 363, 717

Hunter, D. A., Hawley, W. N., \& Gallagher, J. S. 1993, AJ, 106, 1797

Hunter, D. A., \& Hoffman, L. 1999, AJ, 117, 2789

Irwin, M., \& Lewis, J. 2001, New AR, 45, Issue 1-2, 105

Jacoby, G. H. 1989, ApJ, 339, 39

Jacoby, G. H., \& Lesser, M. P. 1981, AJ, 86, 185

Jacoby, G. H., \& Ford, H. 1986, ApJ, 304, 490

Lilly, S. J., Tresse, L., Hammer, F., Crampton, D., \& Le Fevre, O. 1995, ApJ, 455 L108

Madau, P., Pozzetti, L., \& Dickinson, M. 1998, ApJ, 498, 106

Magrini, L., Corradi, R. L. M., Mampaso, A., \& Perinotto, M. 2000, A\&A, 355, 713

Magrini, L., Cardwell, A., Corradi, R. L. M., Mampaso, A., \& Perinotto, M. 2001, A\&A, 367, 498

Mendez, R. H., Kudritzki, R. P., Ciardullo, R., \& Jacoby, G. H. 1993, A\&A, 275, 534

Minniti, D., \& Zijstra, A. A. 1997, AJ, 114, 147

Moles, M., Aparicio, A., \& Masegosa, J. 1990, A\&A, 228, 310

Monet, D., Bird, A., Canzian, B., et al. 1998, USNO-A2.0 (U.S. Naval Observatory, Washington DC)

Oke, J. B. 1990, AJ, 99, 1621

Renzini, A., \& Buzzoni, A. 1986, in Spectral Evolution of Galaxies, ed. C. Chiosi, \& A. Renzini (Reidel), Ap. Space Sci. Lib., 122, 195

Sakai, S., Madore, B. F., \& Freedman, W. L. 1997, AJ, 480, 589

Seaton, M. J. 1979, MNRAS, 187, 73

Strobel, N. V., Hodge, P., \& Kennicutt, R. C. 1991, AJ, 383, 148

Roye, E. W., \& Hunter, D. A. 2000, AJ, 119, 114

Tosi, M., Greggio, L., Marconi, G., \& Focardi, P. 1991, AJ, 102, 951

van den Bergh, S. 2000, in The Galaxies of the Local Group, (Cambridge University Press), 269

Walsh, J. R., Dudziak, G., Minniti, D., \& Zijlstra, A. A. 1997, ApJ, 487, 651

Zijlstra, A. A., \& Pottasch, S. R. 1989, A\&A, 216, 245

Zijlstra, A. A., \& Pottasch, S. R. 1991, A\&A, 243, 478 